\title{
Recognizing Uncommon White Retinal Vasculopathy
}

\section{Paul Varner*}

John J Pershing VAMC, USA

*Corresponding Author: Paul Varner, John J Pershing VAMC, USA.
Received: June 04, 2020

Published: August 25, 2020

(C) All rights are reserved by Paul Varner.

\begin{abstract}
Unusual medical presentations present diagnostic challenges, and correct identification of such conditions represents advanced clinical proficiency. White retinal vessels fall into the category of uncommon ophthalmic findings. This paper reviews presentations of white retinal vasculopathy, the pathophysiology behind those conditions, and the limitations of current terminology.
\end{abstract}

Keywords: Retina; Vasculopathy; Arteries; Arterioles; Veins; Venules; White Vessels; Emboli; Plaque; Sclerosis; Sclerotic Vessel; Attenuation; Sheathing; Ghost Vessel

\section{Introduction}

Health care providers move through a series of steps culminating in the passage of board examinations certifying a basic level of "competency". To achieve this end, initial passive didactic education is integrated with active clinical training and eventually supplanted by immersive patient care. Ultimately, licensure leads to independent practice, yet provider development does not stop there, and the learning curve of clinical knowledge continues, albeit on a flattened trajectory, throughout a career. The medical literature is silent on this accumulation of clinical expertise; however, this author estimates that an additional 5 - 10 years of experience are required after leaving a training program to master the subtle nuances of patient care, to achieve a thorough proficiency required to evaluate the preponderance of cases in a particular practice on any given day, and to gain an appreciation of the anatomical and physiological changes associated with long-term clinical follow-up care.

Because of the extreme variability in pathological manifestations of biological systems, not every single disease entity can be taught to - or even partially absorbed by - students in any training program. Medical trainees are instructed in the recognition of the most common diseases and then - it is hoped - provided a frame- work for application of certain basic medical principles (case history, physical exam, medical decision-making) to unknown patient presentations. Eventually, clinical intuition develops with accumulated experience.

There is no systematic path to advanced clinical knowledge. After structured clinical education is completed, providers are expected to research cases with findings new to them and to consult with colleagues to continue their clinical education - and most do. However, this process continues to be piecemeal - largely dependent upon the caseload of the provider - and the results are haphazard and inconsistent. This gradual attainment of medical knowledge over many years culminates in the attainment of advanced clinical experience but is not attained via a measurable endpoint.

Asymmetric basic clinical experience makes ongoing medical education targeted toward rarer conditions a seemingly insurmountable task as authors and lecturers must be able to relay pertinent information to receptive providers at critical stages during their medical careers. The intent of this paper is to fill one of those demands: to provide a reference for advanced clinical recognition of uncommon retinal vasculopathy that appears "white", to supple- 
ment basic knowledge of pathological states leading to these presentations, and to provide a framework for consideration of these uncommon clinical findings.

\section{Background}

It may be recalled that the main difference between retinal arterioles and venules is anatomical in nature: the arterioles contain 3 layers (tunica intima, tunica media, and tunica adventitia) whereas venules lack both tunica media and tunica adventitia layers [1]. The tangible result of these differences is that arterioles have thicker walls and smaller lumen - the physiological consequence of which is the subtle difference in appearance between retinal arterioles and venules visualized by ophthalmoscopy. The retina is entirely unique in that it is the only place in the body where vasculature can be viewed in vivo, giving practitioners a direct view of human circulation.

In its most basic definition, retinal vasculopathy is a generic term applied to any disease state affecting the retinal vasculature bed - including arteries and arterioles, veins and venules. Overall symptoms of retinal vascular disease follow anatomic location: the closer the affected vessels are to the fovea the more likely visual compromise will result. In some cases, non-macular retinal vascular events may be entirely asymptomatic for patients and, therefore, unrecognized at the time of occurrence.

While many ocular vascular diseases are accompanied by other findings involving retina, choroid, vitreous or optic disk, this review will focus only on those findings that can occur as isolated events - and which appear as "white" changes within the retinal vasculature: primarily, embolization, sheathing, and ghost vessels. It is hoped that a review of these infrequent retinal vasculopathies will supplement clinicians' knowledge by facilitating insight into the underlying processes for specific ophthalmic conditions that currently lack exacting definitions and consensus terminology.

*Note: standard medical definitions discussed below were obtained from online medical dictionaries such as Oxford (https:// www.oxfordreference.com), Medicine Net (https://www.medicinenet.com), or Web MD (https://www.webmd.com).

\section{Embolism}

Embolization is the process by which any object or gas bubble - known as the embolus - or thrombus (a mass of platelets, fibrin and other blood components formed by the active physiological clotting mechanism) is carried through the bloodstream and lodges in a blood vessel. The obstruction itself is the embolism and demarcates a secondary location, distant from the site where the embolus formed. This distinction is important as recognition of the primary site influences medical work-up. Arterial embolism is a mechanical event that results in the sudden, physical interruption of blood flow into and expected sequelae within the target organ.

Retinal arterial embolism indicates the presence of an embolus (or emboli, plural) in a retinal arteriole. It is a phenomenon of retinal arteries only as the vessel diameter within the smaller capillary beds forms an effective physical barrier against emboli lodging within venules. Only within the retinal vasculature is an embolism visible in situ.

Venous thrombosis prevents evacuation of normal blood flow from tissue and is also associated with significant morbidity. The etiology of venous thrombosis remains nebulous, but involves hemostasis, endothelial injury of vein walls, and blood hypercoagulability [2]. The pathophysiology of thrombosis in ophthalmic venous disease is unproved [3], and contrary to some retinal arteriole emboli, ophthalmic thrombi are not visible via ophthalmoscopy.

Both retinal embolism and venous thrombosis are phenomena of aging. Retinal emboli are nine times more likely to be found in a patient 75 years of age or older, than in one younger than 55 [4]. Within the context of suspected retinal venous thrombosis, retinal vein occlusions also have peak incidences in patients over 60 years of age [5].

Eye practitioners are quite familiar with small (focal) emboli: cholesterol, calcific, and platelet-fibrin [6]. Cholesterol emboli are the most common of the focal retinal emboli. These emboli are typically quite small, solitary, involve a single arteriole, and are commonly found at arteriolar bifurcations. Bilateral retinal emboli are rare, although multiple emboli in the same eye are observed more often (Figure 1) [7]. Retinal emboli have a prevalence of roughly $1 \%$ with a 5 - 10 -year incidence also approximately $1 \%$ across population-based studies [8]; however, this data does not address size of the emboli.

Size of the emboli can vary, and larger-sized emboli (even $>1$ disk diameter) are also observed clinically. Therefore, it may be

Citation: Paul Varner. "Recognizing Uncommon White Retinal Vasculopathy". Acta Scientific Ophthalmology 3.9 (2020): 09-22. 


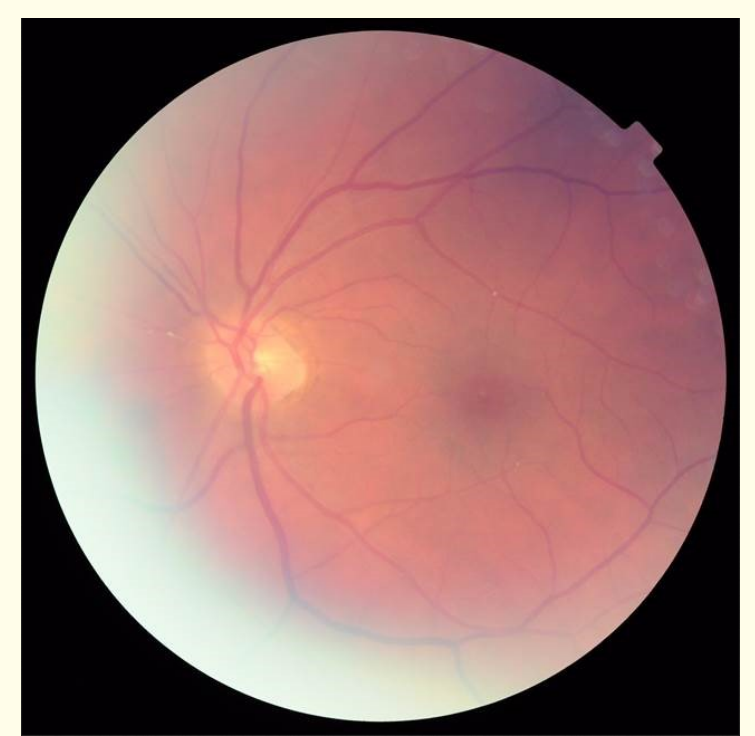

Figure 1: Retinal cholesterol emboli. Three are visible in this photo.

noted that retinal arterial embolism is manifested in two ways: small, "focal" (Figure 1) or much larger emboli (Figure 2). Nomenclature varies for retinal emboli although "Hollenhorst plaque" is readily recognizable, the eponymous term following that author's seminal paper on the topic [9]. It is instructive to note that Hollenhorst included photos of both focal and large emboli in his original series.

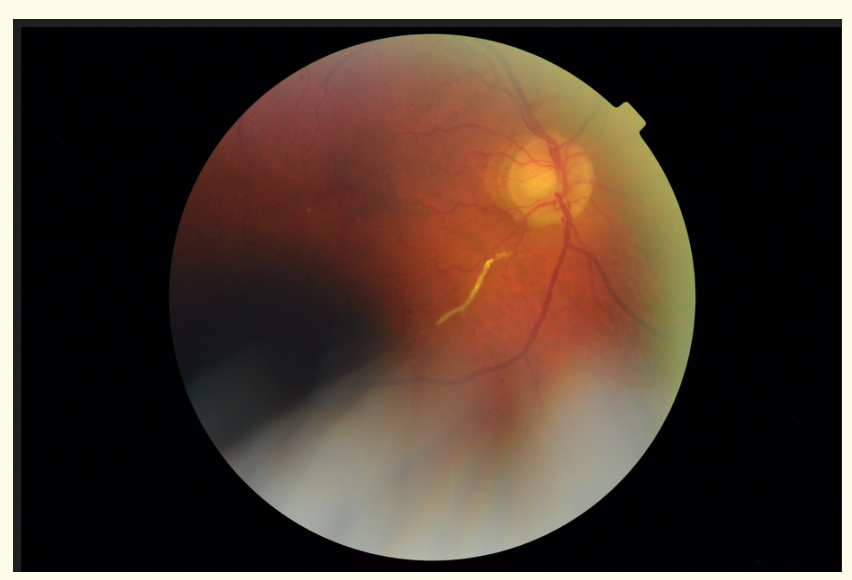

Figure 2: Very large ( $>1 D D$ ) retinal embolus, taken within one year of BRAO. Note partially-refractile nature.
"Plaque" may not be the most descriptive term; however, and in the ophthalmic realm, plaque is also used to describe senescent hyaline changes in the sclera [10] and corneal mucus deposits [11], as well as radioactive Iodine devices used in the treatment of choroidal melanoma [12]. Plaque is defined to be a deposition of material on an arterial wall, associated with atherosclerotic processes. In this context, plaque represents an atheroma. Confusion arises when a fragment of an atheroma breaks off an arterial wall and then becomes a free-floating embolus. Large retinal emboli are also occasionally referred to as "plaques" in the ophthalmic journals.

Whether all large retinal emboli are atherosclerotic plaques is unclear as this has not been studied to date; however, there is currently no specific term to divide focal refractile emboli from much larger, emboli that appear dull with time. Lack of clinical differentiation is perhaps related to the fact that functional outcomes are similar.

Depending on location, retinal emboli can be entirely asymptomatic. When causing symptoms, they may be associated with amaurosis fugax or other visual acuity (VA) and/or visual field (VF) defects. Emboli that result in arterial occlusion are accompanied by non-inflammatory retinal edema corresponding to location of the embolism. Both VA and VF losses are possible, depending on the location of the embolism within the retinal arterial bed (central vs branch), the extent of retinal involvement (only demonstrable by retinal fluorescein angiography, FA), and the longevity of subsequent retinal ischemia. Retinal embolism is typically an isolated event - with recurrence being an exception, rather than the rule. As a final note, emboli may not always be evident in arterial occlusions, even with visible retinal edema.

The appearance of larger emboli can provide some uncertainty and give the impression of a "white" segment of a retinal arteriole. Figure 3 was obtained in the same patient as for figure 2, but two years after the initial event. Note the loss of refractile characteristics for this embolus. Figure 4 represents another large embolism, lodged at an arterial bifurcation, but not refractile in nature. Both photos were incidental findings obtained through diabetic screening protocols (hence the degraded quality of images obtained through non-mydriatic pupils), and the patients were entirely asymptomatic of these retinal findings. 


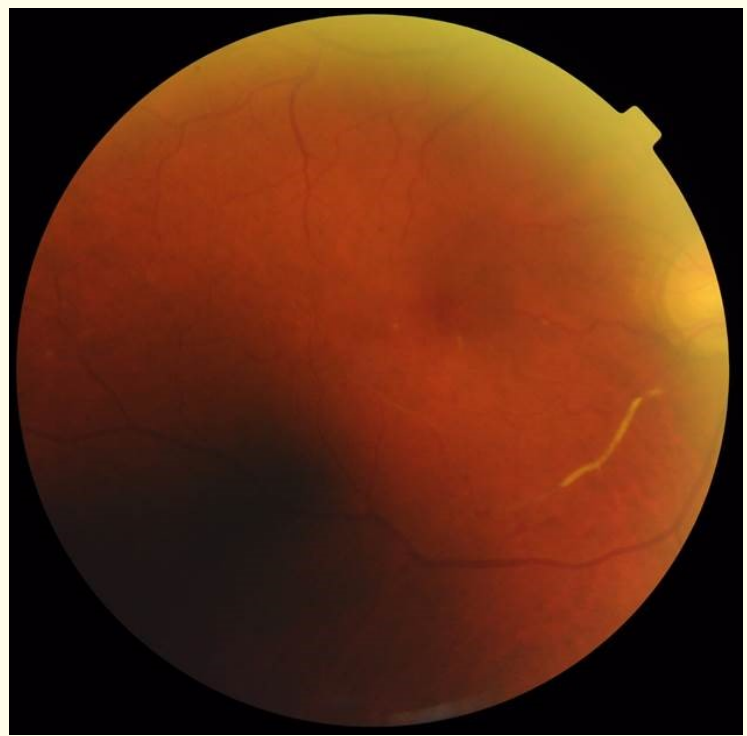

Figure 3: Same embolus as in figure 2; however, with loss of refractile characteristics of embolus. This photo was taken two years after figure 2.

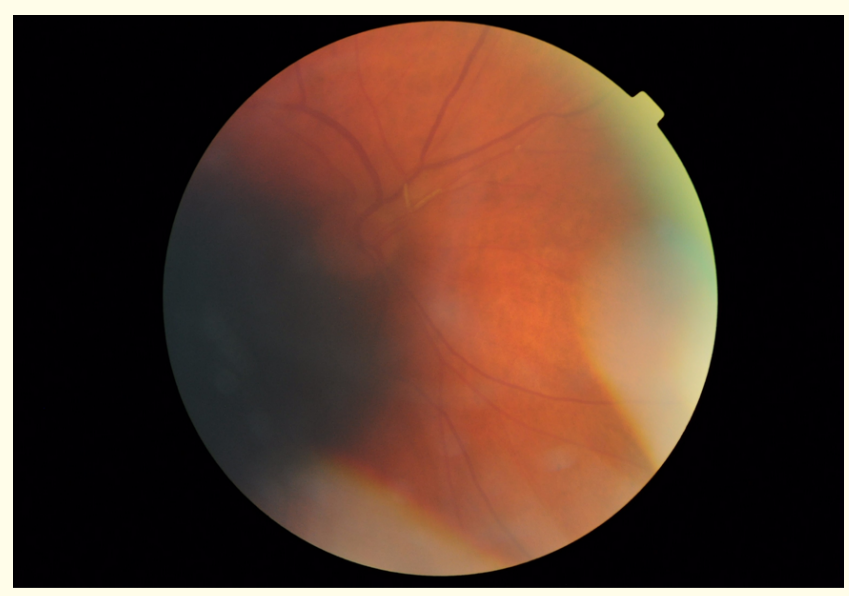

Figure 4: Large bifurcated retinal arterial embolism superior-nasal to optic nerve head.

Focal refractile retinal emboli typically dissipate within a few months; however, large emboli may never completely disappear, and their appearance years after the acute event can be ambigu- ous. The larger the embolus, the less likely it will resolve - even after many years of follow-up, as complete arterial occlusion prevents blood flow to remove embolic material. To confound clinical description further, these larger, sometimes persistent emboli have also been labeled "plaques", "lipid deposition", "sheathing”, or even a "sclerotic" vessel.

Recall, detection of retinal arterial emboli is a secondary event, evident only after embolization (i.e. the primary event) has concluded. Emboli can be visualized within the retinal vasculature during both the acute (in the case of symptomatic arterial occlusions) or the chronic phase (when still visible long after the acute events have resolved in asymptomatic cases).

Such enduring emboli may be generically documented as "retinal arterial embolization". As this is a secondary event, in patients without known medical care standard cardiovascular work-up would be indicated - tailored to discern primary sources: carotid versus cardiac - with or without subsequent anti-platelet therapy, as determined during the cardiovascular risk assessment by a primary care provider.

Further sequelae of retinal arterial embolism (the secondary event) are possible. Tertiary ophthalmic events include central retinal artery occlusion (CRAO) and its self-evident, profound VA loss, or branch retinal artery occlusion (BRAO), possibly affecting VA, and requiring perimetric testing to determine functio laesa via VF losses, or retinal angiography to determine the extent of retinal non-perfusion.

Tertiary cardiovascular events comprise cerebral or cardiac infarction. An asymptomatic retinal embolus in a patient with known cardiovascular disease may benefit only from the addition of daily anti-platelet therapy; however, in the presence of ongoing amaurosis fugax, carotid ultrasound imaging is indicated to rule out carotid artery occlusive disease.

Years after BRAO, VA, peripheral VF scotomata [13] or nerve fiber layer (NFL) defects [14] may be detected. The same morbidities are also noted following branch retinal vein occlusion (BRVO) $[15,16]$. In fact, previously undiagnosed embolic or thrombotic events could be the source of unexplained, localized, peripheral VF defects detected incidentally during subsequent perimetric testing, even many years after the initial event; however, only FA 
could prove extant retinal non-perfusion at that stage [17]. To date there have been no systematic studies of long-term results of retinal non-perfusion via FA or NFL defects through ocular coherence tomography for these retinal events.
13

In fine, retinal embolism (large or small) is a physical obstruction of vasculature. A summary of the characteristics of retinal embolism is given in table 1 , while clinical findings are provided in table 2 .

\begin{tabular}{|c|c|c|c|c|c|c|c|c|c|c|c|c|}
\hline & $\begin{array}{l}\text { Age of } \\
\text { Patient }\end{array}$ & Laterality & $\begin{array}{c}\text { Vascu- } \\
\text { lature } \\
\text { In- } \\
\text { volved }\end{array}$ & $\begin{array}{c}\text { Area } \\
\text { of } \\
\text { Regard }\end{array}$ & $\begin{array}{c}\text { Number } \\
\text { of Vessels } \\
\text { involved }\end{array}$ & $\begin{array}{c}\text { Associ- } \\
\text { ated } \\
\text { Findings }\end{array}$ & $\begin{array}{c}\text { Visual } \\
\text { Symptoms }\end{array}$ & $\begin{array}{l}\text { Chro- } \\
\text { nicity }\end{array}$ & $\begin{array}{l}\text { Anteced- } \\
\text { ent Event }\end{array}$ & $\begin{array}{l}\text { Inflamma- } \\
\text { tion } \\
\text { Involved? }\end{array}$ & $\begin{array}{l}\text { Tran- } \\
\text { sient } \\
\text { Appear- } \\
\text { ance }\end{array}$ & $\begin{array}{c}\text { Rarity } \\
\text { (Inci- } \\
\text { dence) }\end{array}$ \\
\hline \begin{tabular}{|l|} 
Retinal \\
Arterial \\
Emboli- \\
zation \\
\end{tabular} & $>60$ & Unilateral & $\begin{array}{l}\text { Arteri- } \\
\text { oles }\end{array}$ & Small & Single & Few & $+/-$ & Acute & Yes & No & Yes & $1 \%$ \\
\hline \begin{tabular}{|l|} 
Retinal \\
Arterio- \\
sclerosis \\
\end{tabular} & $>60$ & Bilateral & $\begin{array}{l}\text { Arteri- } \\
\text { oles }\end{array}$ & Large & Multiple & Few & - & Chronic & No & No & No & $10 \%$ \\
\hline \begin{tabular}{|l|}
$\begin{array}{l}\text { Vascular } \\
\text { Sheath- } \\
\text { ing }\end{array}$ \\
\end{tabular} & $<50$ & Either & Either & $\begin{array}{l}\text { Focal< } \\
\text { Larger }\end{array}$ & Multiple & Usually & + & Acute & No & Yes & Yes & $\begin{array}{l}\text { Very } \\
\text { Rare }\end{array}$ \\
\hline $\begin{array}{l}\text { Retinal } \\
\text { Ghost } \\
\text { Vessels }\end{array}$ & $>60$ & Unilateral & Either & Either & Few & $\mathrm{Y} / \mathrm{N}$ & $+/-$ & Chronic & Yes & No & No & $\begin{array}{c}\begin{array}{c}\text { Un- } \\
\text { known, }\end{array} \\
\text { But rare }\end{array}$ \\
\hline
\end{tabular}

Table 1: Summary of characteristics for white retinal vessels.

\begin{tabular}{|l|c|c|c|c|c|}
\hline & Etiology & $\begin{array}{c}\text { Visual Acuity } \\
\text { Decrease }\end{array}$ & $\begin{array}{c}\text { Visual Field } \\
\text { Defect }\end{array}$ & $\begin{array}{c}\text { Non-perfusion on } \\
\text { Angiography }\end{array}$ & $\begin{array}{c}\text { Eventual NFL } \\
\text { Defects }\end{array}$ \\
\hline Arterial Embolization & Embolic & $+/-$ & $+/-$ & $+/-$ & $+/-$ \\
\hline Arteriosclerosis & Vascular Remodeling & - & - & - & - \\
\hline Vascular Sheathing & Inflammatory & $+/-$ & $+/-$ & $+/-$ & $+/-$ \\
\hline Ghost Vessels & Ischemic & $+/-$ & + & + & + \\
\hline
\end{tabular}

Table 2: Summary of clinical findings for white retinal vessels.

\section{Retinal arteriosclerosis}

Historically, "sclerosis" of the retinal arterioles encompassed two clinical findings: the "organic thickening of the wall of the arterioles indicated by reflex enhancement and arteriovenous crossing changes" [18] (also reported as "involutionary sclerosis"), and the appearance of white or gray retinal blood arterioles or venules after limited ischemic events [19-21]. However, as "sclerosis" is currently defined as "abnormal hardening of body tissue", and more specifically - arteriosclerosis as the "thickening, hardening, and loss of elasticity of the walls of arteries" the truncated term no longer seems adequately descriptive of late-term pathological processes underlying the ophthalmic clinical presentation that results in the appearance of "white" blood vessels.

Furthermore, retinal arteriosclerosis has been linked to the aging process [22] via replacement of arteriolar wall smooth muscle with collagen [23] - venules are excluded via the anatomic differences mentioned above. This process represents microvascular 
remodeling of arterioles. Well-known multipliers for arteriosclerosis - hyperlipidemia, diabetes, hypertension (HTN) - are considered risk factors for eye diseases as well [24] - but have no acute antecedents. Differentiation of arteriosclerotic from hypertensive retinal disease has long been recognized to be difficult [25], with retinal vascular changes associated with HTN noted in up to $10 \%$ of patients in a population-based study [26] - possibly a confounding, generalized finding among adults $>60$ years of age [25]. As expected of a systemic disease involving the entire human vasculature, retinal arteriosclerosis should be widespread- involving multiple vessels - and to appear over longer segments of the arteries in both eyes - objective retinal findings would not be restricted to a single arteriole in one eye. Figure 5 shows prominent arteriolar light reflexes associated with retinal arteriosclerosis. This subtle finding is hard to discern and essentially appears as prominent arterial light reflexes; however, arteries are not "white" or inflammatory in appearance.
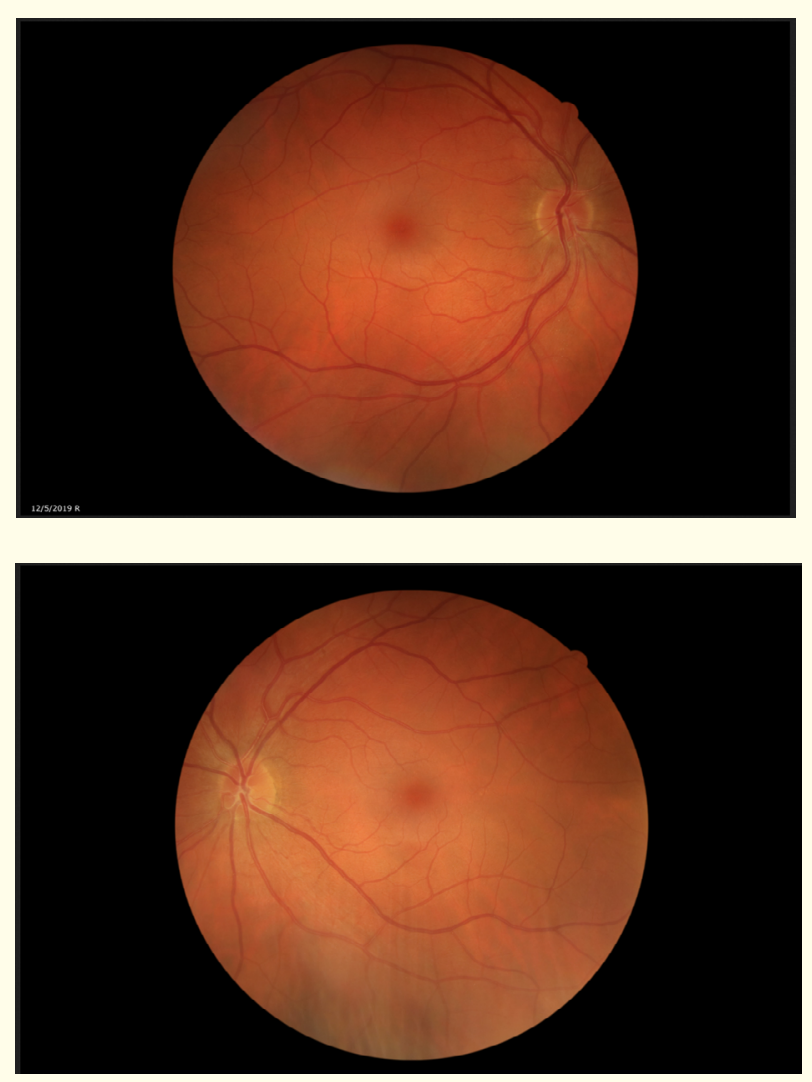

Figure 5: Right and left eyes showing retinal arteriosclerosis of multiple arterioles.
To further complicate case reports and written communications, lengthy areas of arteriolar whitening (several DD), are variously described as "sclerotic", "sheathing" (although not associated with active inflammation, discussed below), or "silver wiring" (in unambiguous cases of advanced systemic hypertension).

Though not systematically studied, "sclerotic" retinal vessels have been incidentally found to correspond with areas of retinal non-perfusion on FA [27], so it seems more likely that "sclerotic" vessels noted after ischemic ophthalmic events are actually referring to "non-perfused" vessels - arterioles or venules - that is, empty vessels with collapsed lumen, or "ghost vessels".

True retinal arteriosclerosis has no acute antecedent event and represents a long-term outcome of chronic, systemic processes. Early, associated retinal findings may include arterio-venular (A/V) nicking or changes in the $\mathrm{A} / \mathrm{V}$ ratio, but these are inconsistently found. Regardless, subjects remain asymptomatic (no expected change in VA or VF, FA changes, or NFL defects), with these findings largely incidental to retinal examination and unexpected to change or resolve over time.

When detected in patients without a known diagnosis of HTN, subsequent medical work-up includes in-office blood pressure check to rule out immediate hypertensive risks and referral to Primary Care for cardiovascular profile.

True retinal arteriosclerosis is the result of vascular remodeling. A summary of the features of retinal arteriosclerosis is offered in table 1 with clinical findings in table 2 .

\section{A note regarding retinal vascular attenuation}

Although not causing a "white" color for retinal vessels, "attenuation" has long been associated with "sclerosis" of retinal arteries $[28,29]$ and continues to be noted for retinal vascular changes associated with systemic hypertension [30]. The underlying mechanism for retinal arteriolar attenuation remains unclear [31], and the utility of this ophthalmic sign outside retinitis pigmentosa [32] is unknown.

Attenuation of only retinal arterioles has been reported following BRAO [33], CRAO [34], and central retinal vein occlusion (CRVO) [35]; whereas combined arteriolar/venular attenuation has been noted following ophthalmic artery occlusion [36] and BRVO [37]. Attenuation is not a universal finding after these events, though. 
Attenuation of retinal arterioles is considered ubiquitous in retinitis pigmentosa [38], and in this clinical circumstance, is felt to be a form of vascular transformation due to decreased metabolic demand of a degenerating retina [39].

In the context of retinal vasculopathy, attenuation is generally thought of as "narrowing of a blood vessel", although in ophthalmoscopic terms this descriptor seems to be used to describe a decrease in the vascular blood column diameter seen clinically as a decreased A/V ratio [40].

Presumably, retinal vascular attenuation represents another generic clinical finding related to ischemic processes, but - by itself - is neither universal nor pathognomonic for any specific retinal vascular disease. Given inconsistencies surrounding the presence of vascular tortuosity [18], it is unclear that this label is clinically useful.

\section{Sheathing}

"Sheathing" of retinal vessels has been used to describe various findings associated with vascular occlusions, embolism, arteriosclerosis, ghost vessels, as well as active inflammation. However, ophthalmic perivascular sheathing has been clearly defined with respect to the underlying pathophysiological process as "a collection of exudation consisting of inflammatory cells around the affected vessels" [41]. The underlying biological response is the break-down of the blood-retina barrier due to active inflammation, a true -itis. This classification is also consistent with the recognition that perivascular sheathing is considered a classic feature of retinal vasculitis [41].

In broad terms, "vasculitis" involves a group of medical disorders that destroy blood vessels via inflammation, and which can affect blood vessels of any type (arteries, veins, capillaries), size or location. More specifically, retinal vasculitis is used to describe conditions involving posterior segment arteries, capillaries, or veins, and which can cause significant vision loss. This descriptive term includes various clinical manifestations including changes in vascular appearance (sheathing, cuffing), leakage, and/or occlusion, often within the setting of posterior uveitis. Retinal vein inflammation is further termed phlebitis, whereas inflammation of retinal arterioles is called arteriolitis - although these terms are infrequently used in the ophthalmic literature.

The exudative response in retinal vasculitis results in the appearance of an elongated "white cuff" around the affected retinal vessel and can affect multiple vessels (arteriolar or venular) in one or both eyes. Retinal vasculitis can occur in the presence of other ischemic findings: retinal hemorrhages, cotton wool spots, extravascular protein deposition, retinal edema, and so on. In the grand scheme of retinal vasculopathy, retinal vasculitis is rare - even within a dedicated uveitis clinic [42] - and there is no clear antecedent event. True retinal vascular sheathing in isolation is probably even more uncommon.

Retinal vasculitis is rarely associated with systemic vasculitides [40]; but in the context of retinal vasculitis associated with multiple sclerosis, there is a break-down of the blood-retina barrier characterized by infiltration of the vessel wall [43], a process unrelated to the demyelinating process [44]. As vascular findings in primary retinal vasculitis and Eales' Disease also have similar appearances [45], it may be presumed that vascular sheathing is a final common pathway found in multiple ophthalmic conditions and not unique to any single disease process.

Both arteries and veins can develop sheaths, even within the same condition [46], and teasing out arteriolar versus venular involvement (i.e. phlebitis vs arteriolitis) can be quite difficult. The clinical relevance of this distinction may be less important than recognition of true inflammatory vascular sheathing, which only occasionally occurs in isolation (i.e. vascular sheathing only, without other associated ophthalmic findings (Figure 6), which can also involve both vascular beds, and which can be a harbinger of posterior uveitis.

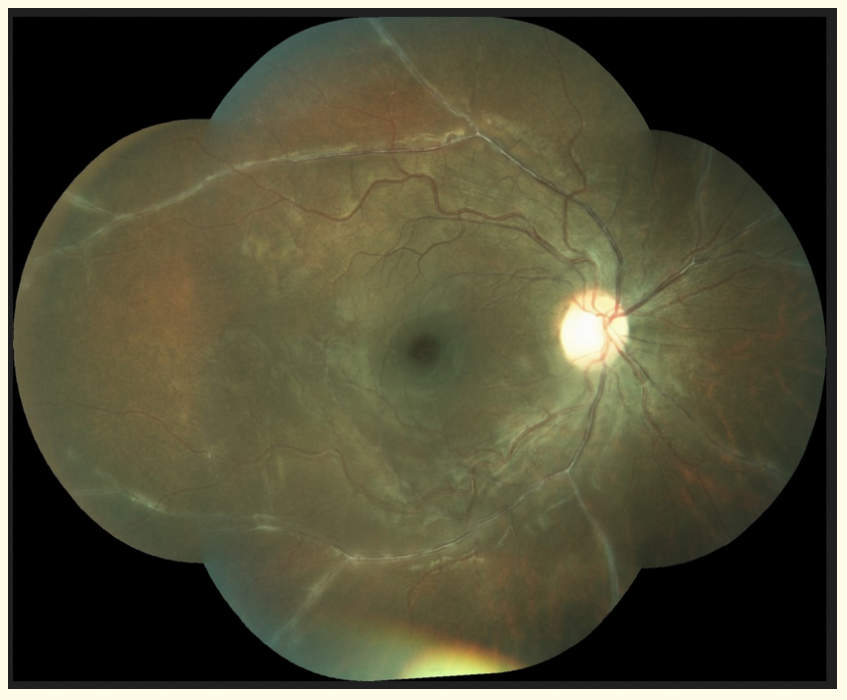

Figure 6: Vascular sheathing. Unpublished Photo courtesy of Mohammad Rafieetary, OD. 
Ophthalmic literature searches indicate that sheathing of retinal veins is more common than arteries. This preponderance of venular involvement is directly related to inflammatory changes at the microscopic level: namely, that inflammatory cell adhesion mainly occurs in post-capillary venules $[47,48]$. In general, the non-specific inflammatory response is more widespread and involves longer sections of multiple vessels. These retinal findings are typically transient in appearance, and when associated with posterior uveitis, more often affect patients younger than 50 years of age $[49,50]$. Reports also seem to suggest that involvement of longer segments of multiple vessels is more common than small isolated areas of regard.

Although sheathing has been used to describe a late ophthalmic finding after both arteriole and venule occlusions, this may be a masquerader as there is no active inflammation years after these events. Hayreh depicts visible sheathing following both BRVO [37] and CRVO [35] - indicating that vascular wall infiltration can persist beyond the acute phase. However, these unusual appearances seem to be exceptions rather than the rule (perhaps necrotic tissue visible after incomplete ghost vessel formation?), and the whitish appearance of vessels after those events most likely represents "ghost vessel" sequelae (to be discussed below). Use of the term sheathing then, is probably best reserved for those acute presentations in which the inflammatory response is associated with active retinal vasculitis, especially in the context of posterior uveitis.

For cases of retinal vasculitis, the acute onset of painless decrease in vision seems to be the most commonly reported visual symptom. Compromise of VA, VF and (presumably) NFL depends on extent and longevity of the vasculitis and secondary retinal non-perfusion.

Vascular sheathing must be thought of in terms of active inflammation. Subsequent work-up follows the differential diagnosis for posterior uveitis and is beyond the scope of this discussion. Clinical traits and findings of retinal vascular sheathing are provided in table 1 and 2 .

\section{Ghost vessels}

Over time in the ophthalmic literature, multiple retinal vascular presentations have been characterized by the appearance of strand-like, retinal blood vessels that are white in color, but are the result of disparate - embolic, arteriosclerotic, and sheathing - pro- cesses. In reviewing these cases, though, it seems most likely that many of these atypical vascular appearances are the result of ghost vessel formation - sequelae of an ischemic event.

This finding is not restricted to retinal vasculopathy, as ghost vessels are also occasionally - but inconsistently - mentioned in cases of vascular non-perfusion detected via doppler ultrasonography and non-ophthalmic angiograms (CT angiography, MRA), indicating that similar processes also occur within systemic vasculopathy. For ophthalmic disease this phrase is more frequently applied to keratopathy but seems to be the more accurate descriptor for certain retinal vascular events.

In the retina, ghost vessel development represents a final common pathway for several hypoxic processes and - consistent with demographics of ischemic retinal diseases - are most evident in older patients. Though not a common outcome, any hypoxic retinal process could result in the eventual appearance of a "white" vessel - due to the accumulation of material within the lumen [51] and subsequent lack of blood flow through the vessel lumen, when followed by resultant vascular wall ischemia. Microscopically, this may be surmised to be the result of vascular endothelium cell death [52]. This has been secondarily described in conditions affecting both arterioles [53] and venules [54].

Ghost vessel development can occur in retinal arterioles after vascular occlusion (BRAO, Figure 7), following Pan-Retinal Photocoagulation (PRP, Figure 8), or in association with retinal lattice degeneration; or in venules following presumed vascular thrombosis (BRVO, Figure 9). In either vascular bed, ghost vessel occurrence seems to be the result of vessel wall hypoxia and/or collapse with loss of normal lumen and the subsequent non-perfusion that results in ischemic vascular walls. The culmination of these events is the appearance of a white vessel - what has historically been referred to as a "sclerotic" vessel, but no longer seems to adequate describe the underlying pathogenesis. As this process is not acutely inflammatory, labeling such findings as "sheathing" is equally inaccurate, albeit still found in many references.

From a microvascular standpoint: true sclerotic processes (i.e. abnormal hardening of body tissue) must be confined to retinal arterioles and not veins due to the anatomical differences between retinal arteries and veins as discussed above and should affect all arterioles indiscriminately (as in HTN retinopathy); however, these

Citation: Paul Varner. "Recognizing Uncommon White Retinal Vasculopathy". Acta Scientific Ophthalmology 3.9 (2020): 09-22. 


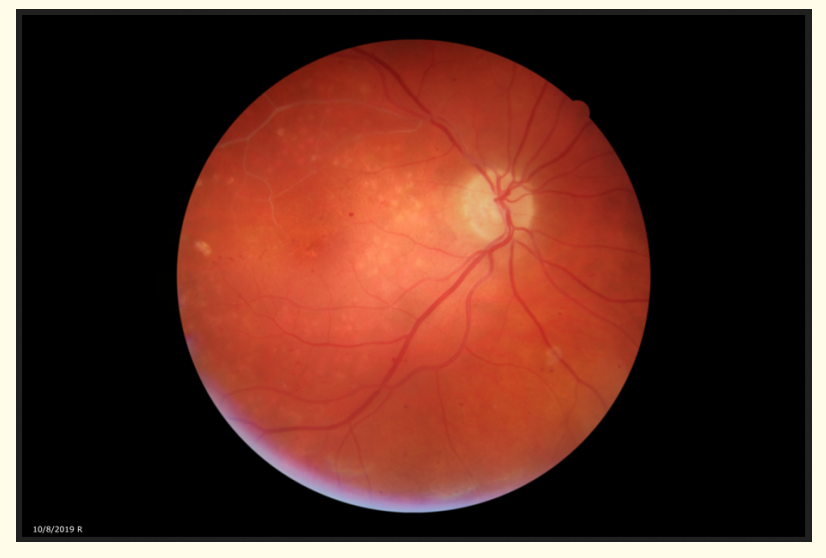

Figure 7: Superior-temporal ghost vessels in arterioles following documented BRAO.

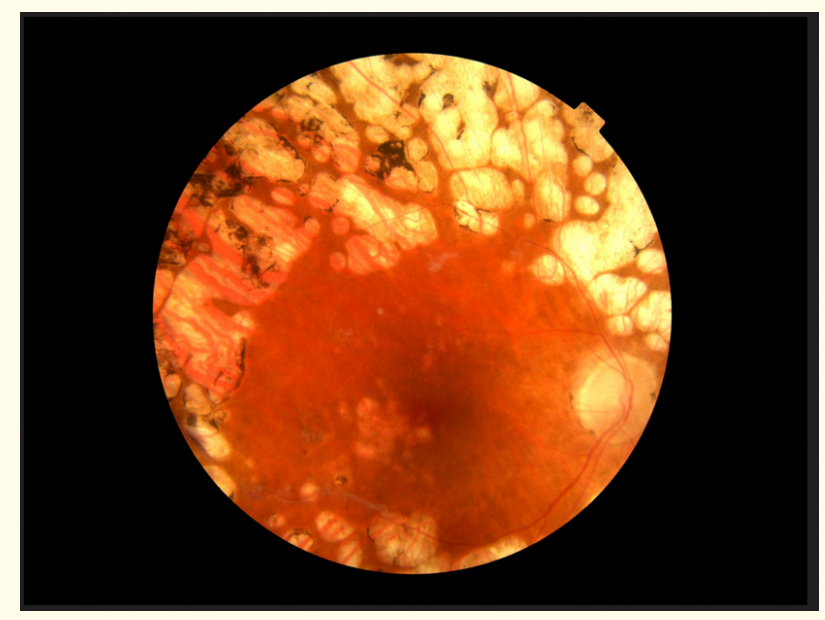

Figure 8: Inferior-temporal ghost vessel in venule following extensive pan-retinal photocoagulation.

white vessels are also seen in venules. Obviously the "replacement of the muscular layer" [55] can only occur in arterioles as the venules do not have the analogous tunica. So, if the anatomy of venules precludes "sclerosis", what causes the appearance of a ghost vessel after BRVO - a vasculopathy confined to venules? As FA and optical coherence tomography (OCT) demonstrate retinal non-perfusion following BRVO [56,57], then it must be concluded that complete occlusion of the vascular lumen eventually results in

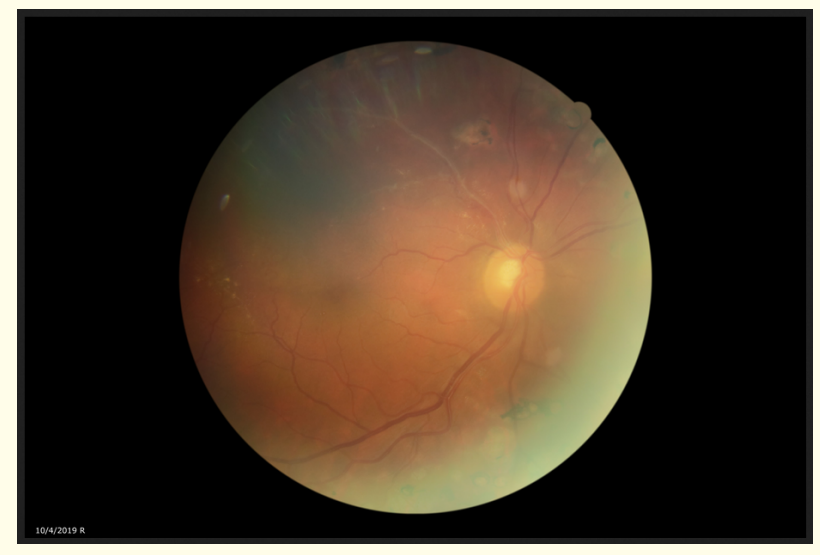

Figure 9: Superior-temporal ghost vessels in venules following documented BRVO.

hypoxia of the venule itself with the subsequent change in color of the necrotic vein.

The number of ghost vessels present is ostensibly dependent upon the extent and longevity of retinal non-perfusion - though not all retinal ischemic events result in total death of the vascular basement membranes [58]. Ghost vessel formation is visible clinically as a longer segment (usually $>1 \mathrm{DD}$ ) of white vasculature within a single vessel (more common, figure 8 and 9) or as several geographically adjacent vessels (rarer, figure 7). More widespread hypoxia would yield more diffuse retinal vasculopathy, indicated by the extent of changes in vascular appearance visible by ophthalmoscopy. - a process that likely occurs over a period of years, that does not disappear with time (i.e. is permanent), and that would require FA to confirm ongoing retinal non-perfusion. Additionally, due to the nature of the antecedent event, the appearance of retinal ghost vessels would not be expected to be a bilateral phenomenon.

Ischemic events culminating in the formation of ghost vessels generally result in VF defects corresponding to the areas of retinal non-perfusion as demonstrated by FA. Central vision may be decreased depending on proximity of the preceding event to fovea; but for non-macular involvement, patients may have been asymptomatic at the time of the event (none of the cases represented in figure 7-9 suffered VA loss). In the absence of ghost vessel formation and decreased VA, the antecedent event may be difficult to dis- 
cern years later - especially in the cases of prior vascular occlusion [59]. However, it should be pointed out that these events may account for otherwise unexplained regional VF defects detected on perimetry, regions of FA non-perfusion, or sectoral NFL defects detected years later. Retinal ghost vessels have not been systematically studied or widely reported.

The finding of a retinal ghost vessel indicates a remote ischemic event. Due to the late discovery of ghost vessels, no immediate medical work-up is indicated. Summaries of the characteristics and visual symptoms and signs associated with retinal ghost vessels are provided in table 1 and 2 .

\section{Completing the differential diagnosis}

Three other ophthalmic conditions involve visualization of multiple white retinal arterioles.

The retinal vasculopathy associated with lipemia retinalis (Figure 10 ) has been known since the $19^{\text {th }}$ century and is most often an incidental finding that rarely affects vision [60]. White or pink vascular changes occur throughout the ocular fundus, involves arterioles and venules [61], is due to excessive lipid concentration in the blood plasma, and is entirely reversible within a relatively short period of time [62]. Lipemia retinalis is directly related to hypertriglyceridemia - associated with triglyceride levels $>2,000$ $\mathrm{mg} / \mathrm{dL}$ [61]. Due to the risk of acute pancreatitis, patients are often admitted for plasmapheresis, and in the rare instance of associated visual compromise, typically recover vision after reduction of blood triglyceride levels to $<200 \mathrm{mg} / \mathrm{dL}$ [63]. The epidemiology of lipemia retinalis is largely unknown, but hypertriglyceridemia is more likely in the 40-59 age group [61].

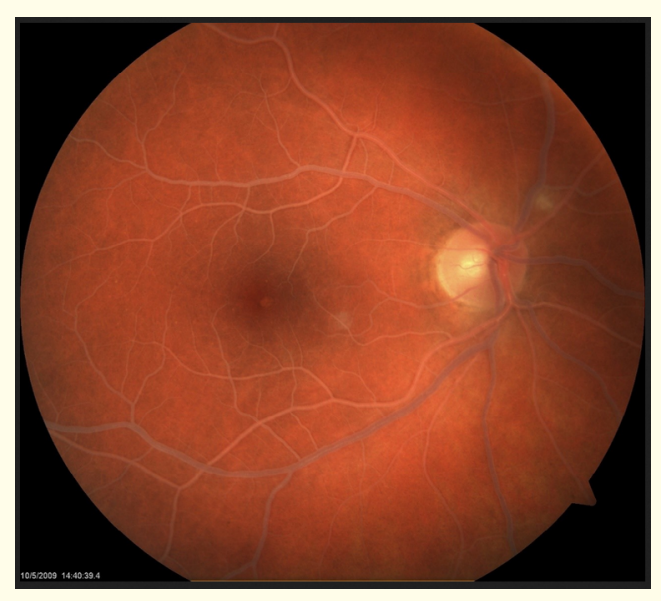

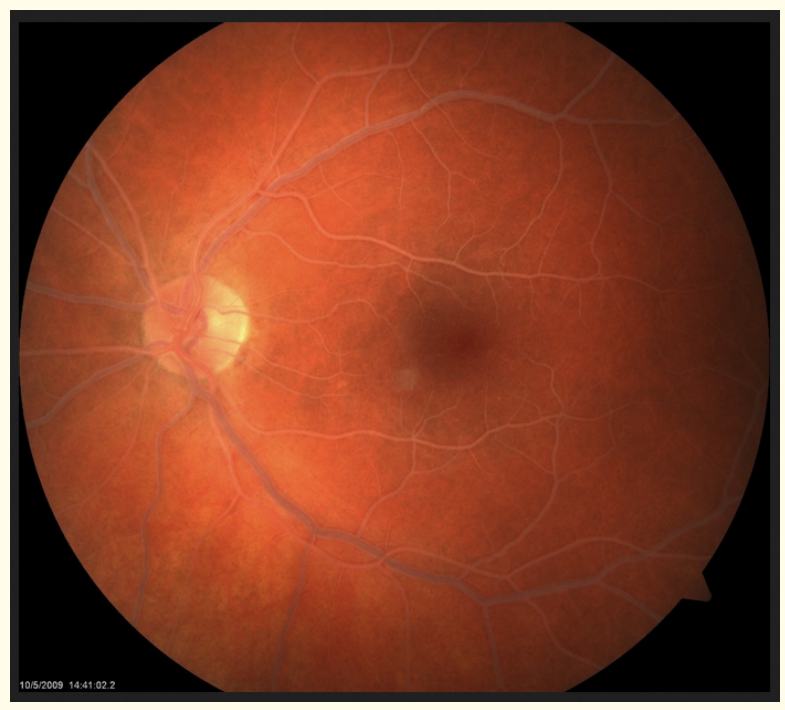

Figure 10: Right and left eyes demonstrating Lipemia Retinalis. Photo courtesy of Matt Horton, OD.

Originally published in Optometry. 2011;82:475-480.

Ophthalmic signs unique to malarial retinopathy in very ill children were only first described in 1993 [64]. In addition to retinal whitening and hemorrhages resembling Roth spots, orange or white discoloration of peripheral retinal vessels occurs in $20 \%$ of cases [65] (Figure 11). The source for this vasculopathy is related to dehemoglobinization associated with the Plasmodium infection in advanced cases of advanced cerebral malaria [66], and can affect arterioles, capillaries and venules [67]. The appearance is reversible [68] and in children who survive, visual acuity seems unaffected [69].

Finally, in addition to recurrent conjunctivitis and band keratopathy, white arterioles have been noted to be a complication of hypercalcemia and renal failure. ${ }^{70}$ The normal serum calcium level is $8.5-10.5 \mathrm{mg} / \mathrm{dL}$ (ranges vary between laboratories), and persistently elevated levels can cause arteriolar calcification due to deposition of calcium in walls of the tunica media. This finding can potentially result in ophthalmic ischemic events and subsequent vision loss. Involvement seems to be widespread within the arteriolar bed and long-term follow-up is unclear - this unique presentation has been reported only once. 


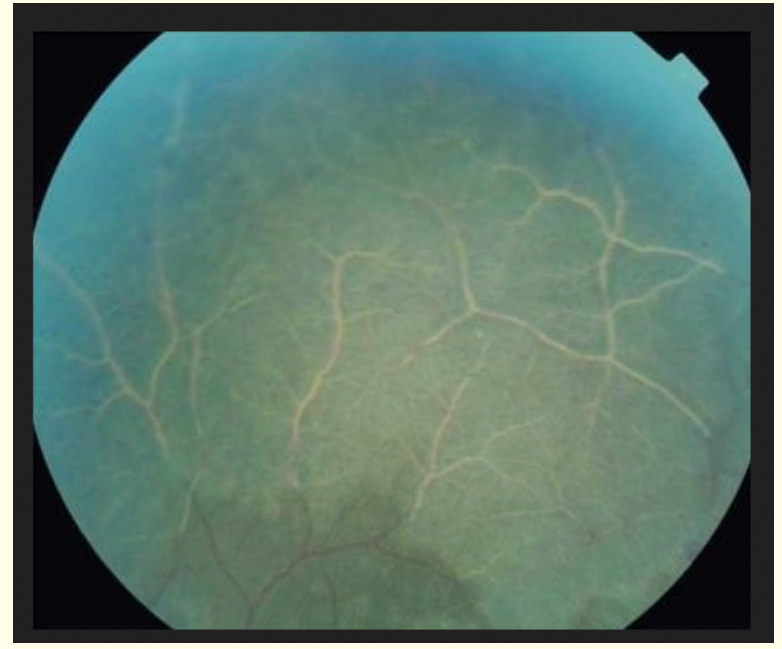

Figure 11: White vessels within area of retinal whitening in malarial retinopathy.

Photo courtesy of Nicholas Beare, MD.

Originally published in The American Journal of Tropical Medicine and Hygiene. 2006;75:790-797.

Due to the extreme nature of these three conditions, they are not included in the summaries of white vessel vasculopathy enumerated in table 1 and 2 .

\section{Conclusion}

The accumulation of sophisticated clinical experience involves mastering the recognition of rare, subtler clinical presentations and is only possible through years of direct and ongoing clinical experience in addition to diligent self-education by providers. Correct recognition and discernment of "white" retinal blood vessels falls into this area of advanced clinical skills attainment. A deeper understanding of the underlying physical processes associated with white retinal blood vessel formation will improve diagnostic accuracy, stratify associated health risk factors and improve patient care. This paper is intended to enhance advanced clinical knowledge in this arena.

\section{Disclosures}

The views expressed in this article are those of the author and do not necessarily represent the position of the Department of Veterans Affairs. The author reports no proprietary or commercial conflicts of interest for any product or concept discussed in this article.
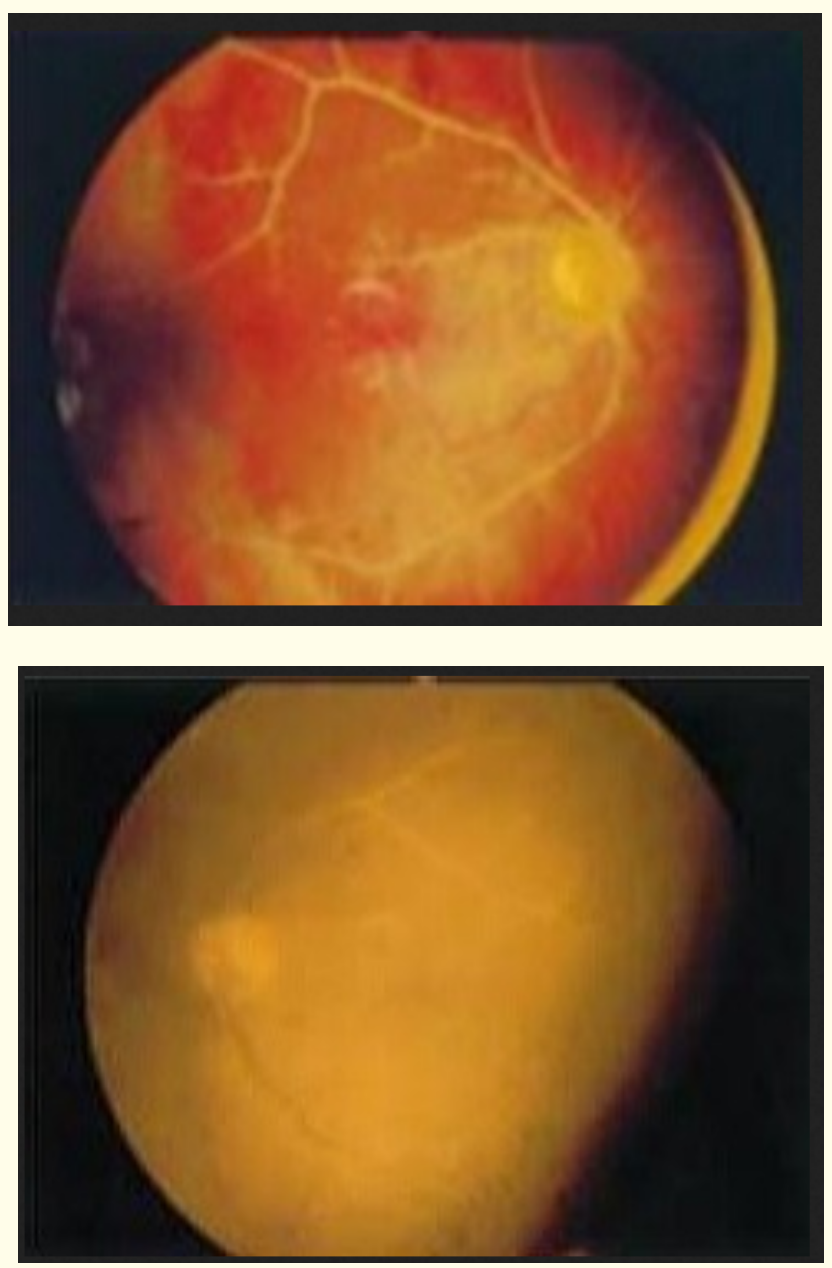

Figure 12: Bilateral retinal arteriolar calcification.

Photo courtesy of Martin Snead, MD.

Originally published in British Journal of Ophthalmology. 2002;86:1063-1069.

\section{Acknowledgements}

The author would like to thank Antonia Varner for her assistance in the design of the tables. Unless otherwise specified, retinal photographs were obtained at the author's facility.

\section{Bibliography}

1. Joshi VD., et al. "Cardiovascular System". In: Anatomy and Physiology for Nursing and Healthcare Students". $3^{\text {rd }}$ edition. New Delhi: BT Publications Pvt Ltd (2006): 163-210. 
2. Myers Jr DD. "Pathophysiology of venous thrombosis". Phlebology 30 (2015): 7-13.

3. Fraenkl, SA., et al. "Retinal vein occlusions: the potential impact of a dysregulation of the retinal veins". EPMA Journal 1 (2010): 253-261.

4. Klein R., et al. "Retinal emboli and stroke: the Beaver Dam Eye Study". Archives of Ophthalmology 117 (1999): 1063-1068.

5. Song P., et al. "Global epidemiology of retinal vein occlusion: a systematic review and meta-analysis of prevalence, incidence, and risk factors". Journal of Global Health 9 (2019): 010427.

6. Wong TY and Klein R. "Retinal arteriolar emboli: epidemiology and risk of stroke". Current Opinion on Ophthalmology 13 (2002): 142-146.

7. Cho KH., et al. "The characteristics of retinal emboli and its association with vascular reperfusion in retinal artery occlusion". Investigative Ophthalmology and Visual Science 57 (2016): 4589-4598.

8. Kim MJ and Fong DS. "Retinal emboli". World Journal of Ophthalmology 4 (2014): 124-129.

9. Hollenhorst RW. "Significance of bright plaques in the retinal arterioles". Transactions of the American Ophthalmological Society 59 (1961): 252-273.

10. Horowitz S., et al. "Prevalence and factors associated with scleral hyaline plaque: clinical study of older adults in southeastern Brazil". Clinical Ophthalmology 9 (2015): 1187-1193.

11. Fraunfelder FT., et al. "Corneal mucus plaques". American Journal of Ophthalmology 83 (1977): 191-197.

12. Perez BA., et al. "Uveal melanoma treated with iodine- 125 episcleral plaque: an anlysis of dose on disease control and visual outcome". International Journal of Radiation Oncology Biology Physics 89 (2014): 127-136.

13. Hayreh SS., et al. "Branch retinal artery occlusion: natural history of visual outcome. Ophthalmology 116 (2009): 11881194.

14. Leung CKS., et al. "In vivo measurements of macular and nerve fibre layer thickness in retinal arterial occlusion". Eye 21 (2007): 1464-1468.
15. Hayreh SS., et al. "Branch retinal vein occlusion: natural history of visual outcome”. JAMA Ophthalmology 132 (2014): 13-22.

16. Kim C-S., et al. "Sectoral retinal nerve fiber layer thinning in branch retinal vein occlusion". Retina 34 (2014): 525-530.

17. Gass, JDM. "A fluorescein angiographic study of macular dysfunction secondary to retinal vascular disease: I. Embolic retinal artery obstruction". Archives of Ophthalmology 80 (1968): 535-549.

18. Stokoe NL and Turner RWD. "Normal retinal vascular pattern: arteriovenous ratio as a measure of arterial caliber". British Journal of Ophthalmology 50 (1966): 21-40.

19. Jensen VA. "Clinical studies of tributary vein thrombosis". Acta Ophthalmology 14 (1936): 23-62.

20. Wise GN. "Arteriosclerosis secondary to retinal vein obstruction". Transactions of the American Ophthalmological Society 26 (1958): 361-380.

21. Dark AJ and Rizk SN. "Progressive focal sclerosis of retinal arteries: a sequel to impaction of cholesterol emboli". British Medical Journal 1.5535 (1967): 270-273.

22. Grossniklaus HE., et al. "Anatomic alterations in aging and agerelated disease of the eye". Investigative Ophthalmology and Visual Science 54 (2013): ORSF23-27.

23. Michelson G., et al. "Morphometric age-related evaluation of small retinal vessels by scanning laser doppler flowmetry: determination of a vessel wall index". Retina 27 (2007): 490-498.

24. Flammer J., et al. "The eye and the heart". European Heart Journal 34 (2013): 1270-1278.

25. Leishman R. "The eye in general vascular disease: hypertension and arteriosclerosis". British Journal of Ophthalmology 41 (1957): 641-701.

26. Wong TY., et al. "The prevalence and risk factors of retinal microvascular abnormalities in older persons: The Cardiovascular Health Study". Ophthalmology 110 (2003): 658-666.

27. Callaway NF and Mruthyunjaya P. "Widefield imaging of retinal and choroidal tumors". International Journal of Retina and Vitreous 5 (2019): 49. 
28. Pines N. "Sclerosis of the Retinal Vessels". British Journal of Ophthalmology 13 (1929): 161-199.

29. O’Donoghue WD. "Retinal Vascular Sclerosis". Irish Journal of Medical Science 20 (1945): 214-230.

30. Henderson AD., et al. "Hypertension-related eye abnormalities and the risk of stroke". Reviews in Neurological Diseases 8 (2011): 1-9.

31. Nakagawa S., et al. "Association of retinal vessel attenuation with visual function in eyes with retinitis pigmentosa". Clinical Ophthalmology 8 (2014): 1487-1493.

32. Ma Y., et al. "Quantitative analysis of retinal vessel attenuation in eyes with retinitis pigmentosa". Investigative Ophthalmology and Visual Science 53 (2012): 4306-4314.

33. Hayreh SS. "Acute retinal arterial occlusive disorders". Progress in Retinal and Eye Research 30 (2011): 359-394.

34. Hayreh SS and Zimmerman MB. "Fundus changes in central retinal artery occlusion". Retina 27 (2007): 276-289.

35. Hayreh SS and Zimmerman MB. "Fundus changes in central retinal vein occlusion”. Retina 35 (2015): 29-45.

36. Sachdeva V., et al. "Spontaneous ophthalmic artery occlusion in children due to hyperhomocysteinemia". Oman Journal of Ophthalmology 8 (2015): 122-124.

37. Hayreh SS nad Zimmerman MB. Fundus changes in branch retinal vein occlusion". Retina 35 (2015): 1016-1027.

38. Hartong DT., et al. "Retinitis pigmentosa". Lancet 368 (2006): 1795-1809.

39. Grunwald JE., et al. "Retinal hemodynamics in retinitis pigmentosa". American Journal of Ophthalmology 122 (1996):502508.

40. Liew G., et al. "Measurement of retinal vascular caliber: issues and alternatives to using the arteriole to venule ratio". Investigative Ophthalmology and Visual Science 48 (2007): 52-57.

41. Do Bk and Giovinazzo J. "Retinal Vasculitis". In: Advances in Ophthalmology and Optometry. Yanoff M, Editor in Chief. Philadelphia: Elsevier Health Sciences 2016. E-Book (2016).
42. Rosenbaum JT., et al. "Patients with retinal vasculitis rarely suffer from systemic vasculitis". Seminars in Arthritis and Rheumatism 41 (2012): 859-865.

43. Birch MK., et al. "Retinal venous sheathing and the blood-retinal-barrier in multiple sclerosis". Archives of Ophthalmology 114 (1996): 34-39.

44. Rucker CW. "Sheathing of the retinal veins in multiple sclerosis". JAMA 127 (1945): 970-973.

45. Crawford $\mathrm{Cm}$ and Hwang Y. "Primary retinal vasculitis vs Eales' disease". International Journal of Open Access Ophthalmology 1 (2016): 3 .

46. Abu El-Sharar Am., et al. "Retinal vasculitis". Ocular Immunology and Inflammation 13 (2005): 415-433.

47. Jackson WF. “Microcirculation”. In: Muscle: Fundamental Biology and Mechanisms of Disease New York: Elsevier 1 (2012): 1197-1206.

48. Sumagin R and Sarelius IH. "Emerging understanding of roles for arterioles in inflammation". Microcirculation 20 (2013): 679-692.

49. Bajwa A., et al. "Epidemiology of uveitis in the Mid-Atlantic United States". Clinical Ophthalmology 9 (2015): 889-901.

50. Engelhard SB., et al. "Intermediate uveitis, posterior uveitis, and panuveitis in the Mid-Atlantic USA". Clinical Ophthalmology 9 (2015): 1549-1555.

51. Bek T and Ledet T. "Vascular occlusion in diabetic retinopathy: a qualitative and quantitative histopathological study". Acta Ophthalmologica Scandinavica 74 (1996): 36-40.

52. Vila N., et al. "Usefulness of discarded vitreous samples from routine vitrectomy". Journal of Ophthalmology 2016 (2016): 2380764.

53. Rehmani A., et al. "Unilateral vascular occlusive disease without vision loss in neurofibromatosis type 1". Clinical and Experimental Optometry 30 (2019): 112-114.

54. Kang HM., et al. "Focal lamina cribrosa defects and significant peripapillary choroidal thinning in patients with unilateral branch vein occlusion". PLOS ONE 15 (2020): e0230293. 
55. Frank RN. "The Eye in Hypertension”. In: Hypertension Primer, 4th ed. Izzo JL, Sica DA, Black HR, eds. Philadelphia: Lippincott Williams and Wilkins (2008): 226-228.

56. Aghdam KA., et al. "Peripheral retinal non-perfusion and treatment response in branch retinal vein occlusion". International Journal of Ophthalmology 9 (2016): 858-862.

57. Shiraki A., et al. "Evaluation of retinal nonperfusion in branch retinal vein occlusion using wide-field optical coherence tomography angiography". Acta Ophthalmology 97 (2019): e913-e918.

58. Powner MB., et al. "Evaluation of nonperfused retinal vessels in ischemic retinopathy". Investigative Ophthalmology and Visual Science 57 (2016): 5031-5037.

59. Sutherland JE and Steahly LP. "Selected Disorders of the Eye". In: Family Medicine: Principles and Practice, 4th ed. New York: Springer-Verlag. (1994): 542-549.

60. Shannon CEG and Mohler HK. "Lipemia retinalis in a diabetic". Transactions of the American Ophthalmological Society 27 (1929): 194-203.

61. Mishra C and Tripathy K. "Lipemia Retinalis". Treasure Island (FL): StatPearls Publishing 2020. E-Book.

62. Akritidis N., et al. "White retinal vessels". Postgraduate Medical Journal 81 (955) (2005): 341.

63. Stifter E., et al. "Complete recovery of lipemia retinalis with visual loss after early plasmapheresis". American Journal of Case Reports 9 (2008): 435-438.

64. Lewallen S., et al. "Ocular fundus findings in Malawian children with cerebral malaria”. Ophthalmology 100 (1993): 857 861.

65. Hirneiss C., et al. "Ocular changes in tropical malaria with cerebral involvement - results from the Blantyre Malaria Project”. Klin Monbl Augenheilkd 222 (2005): 704-708.

66. Lewallen S., et al. "Clinical-histopathological correlation of the abnormal retinal vessels in cerebral malaria". Archives of Ophthalmology 118 (2000): 924-928.

67. Barrera V., et al. "Neurovascular sequestration in paediatric
P. Falciparum malaria is visible clinically in the retina". Elife (2018): 7:pii: e32208.

68. Sithole HL. "A review of malarial retinopathy in severe malaria”. South African Optometrist 70 (2011): 129-135.

69. Beare NAV., et al. "Visual outcomes in children in Malawi following retinopathy of severe malaria". British Journal of Ophthalmology 88 (2004): 321-324.

70. Patel DV., et al. "Retinal arteriolar calcification in a patient with chronic renal failure". British Journal of Ophthalmology 86 (2002): 1063-1069.

\section{Assets from publication with us}

- Prompt Acknowledgement after receiving the article

- Thorough Double blinded peer review

- Rapid Publication

- Issue of Publication Certificate

- High visibility of your Published work

Website: www.actascientific.com/

Submit Article: www.actascientific.com/submission.php

Email us: editor@actascientific.com

Contact us: +919182824667 